\title{
SEARCH AND IDENTIFICATION OF SPERMATOZOA AND SPERMATIDS IN THE EJACULATE OF NON-OBSTRUCTIVE AZOOSPERMIC PATIENTS
}

\author{
ODIVAL TIMM JR, AGNALDO P. CEDENHO, DEBORAH M. SPAINE, MARCIA H.P. \\ BUTTIGNOL, RENATO FRAIETTA, VALDEMAR ORTIZ, MIGUEL SROUGI \\ Laboratory of Human Reproduction, Division of Urology, Paulista School of Medicine, Federal \\ University of São Paulo, UNIFESP, São Paulo, Brazil
}

\begin{abstract}
Objective: To search and to identify spermatozoa and spermatids, present in the ejaculate of non-obstructive azoospermic patients.

Materials and Methods: 27 patients, aged between 18 and 48 years, with initial diagnosis compatible with non-obstructive azoospermia, underwent up to 3 seminal samples, with assessment of macroscopic and microscopic parameters differentiated for each sample. In the first sample, $5 \mu \mathrm{L}$ of semen were analyzed in a Horwell chamber in order to assess the presence or absence of spermatozoa. The procedure was repeated with 2 other aliquots. In the absence of spermatozoa, the entire sample was transferred to a conic tube and following centrifugation the sediment was freshly analyzed. The second seminal sample was collected only when no spermatozoa were found in the first sample and the research was performed in the same way. In cases where spermatozoa were not seen, the sample was centrifuged and the obtained sediment was stained by the panoptic method and observed under common light microscopy (1250X). The third seminal sample was collected only in cases when patients had not shown spermatozoa in the first and second seminal samples.

Results: 4/27 (14.8\%) patients presented spermatozoa in the first seminal sample and 6/23 (26.1\%), in the second seminal sample. No spermatozoa were seen in the third sample, however, $11 /$ $17(64.7 \%)$ presented spermatids.

Conclusion: In clinical situations where the initial diagnosis is non-obstructive azoospermia, one single routine seminal analysis is not enough to confirm this diagnosis and the analysis of the centrifuged sediment can have relevant clinical consequences. Among patients considered non-obstructive azoospermic, when duly assessed, $37 \%$ presented spermatozoa and $64.7 \%$, spermatids.
\end{abstract}

Key words: male infertility; azoospermia; sperm capacitation; spermatozoa; spermatid Int Braz J Urol. 2005; 31: 42-48

\section{INTRODUCTION}

The male factor in conjugal infertility is expressive and, thus, must receive close attention from all health professionals who develop activities in the human reproduction field. Alone it is responsible for $30 \%$ of causes of conjugal infertility and associated with the female factor in more than $20 \%$, the male component has been targeted in many studies and paradigm changes (1).

For a long time, the search and identification of germ cells in their various developmental stages in ejaculated semen have received little or no attention. Reasons for this fact are numerous and we can mention that in fresh exam of ejaculated semen, the identification of such cells is not an easy process and 
demands great expertise of the examiner, is time-consuming and, above all, until recently it did not change prognosis in terms of treatment. For all these reasons, these cells often appear in reports under the generic term of round cells, with no other connotation. The fresh analysis of such cells does not allow accurately distinguishing round spermatids from leukocytes neither from other germ cells that can appear in the semen. However, with the introduction and increasing indications intracytoplasmic sperm injection in human oocytes, the search of germ cells in the ejaculate, especially in non-obstructive azoospermic patients, has gained major importance. Firstly, the presence of such cells can allow the injection of this haploid material in oocytes from this patient's partner in an in vitro fertilization program, which has already enabled the birth of normal children. The first term pregnancy achieved with the use of round spermatids occurred in 1995 (2,3). Secondly, with the technical advance that we have witnessed in the last few years, the identification and isolation of such cells could enable in-vitro culturing, propelling them to more developed stages, that is, round spermatids becoming elongated spermatids with more resilient DNA bands and less subjected to fragmentation during chromosomal pairing (4). In the near future, the characterization of these cells in the ejaculate can allow the use of genic therapy for correcting eventual defects in spermatogenesis.

When considered, these new perspectives warrant the current concern in exhaustively search for the presence of spermatozoa or young elements of the germ lineage, in the ejaculate of non-obstructive azoospermic patients. This work aims to search and identify spermatozoa and spermatids in the ejaculate of patients classified as non-obstructive azoospermic.

\section{MATERIALS AND METHODS}

This study was prospectively performed in the period from January to December 2002 and was approved by the institutional Research Ethics Committee.

Twenty-seven patients aged between 18 and 48 years, with initial diagnosis compatible with nonobstructive azoospermia, were duly enrolled and at- tended. The inclusion criteria for this study were patient classified as non-obstructive azoospermic, with no relevant antecedents concerning the reproductive system, and the exclusion criterion was non-obstructive azoospermic patient with leukocytospermia (> $1.0 \times 10^{6}$ neutrophils $\left./ \mathrm{mL}\right)(5)$.

Testicular volume was measured using the Prader orchidometer. Hormonal assessment was performed through dosing of follicle stimulating hormone (FSH), in serum, by the immunometric technique (Immulite, DPC, USA) (normal $=0.7$ to $11.1 \mathrm{mIU} /$ $\mathrm{mL})$.

All patients participating in this research underwent up to 3 seminal analyses (Figure-1). In an area annex to the laboratory, patients collected semen samples through masturbation, in one-way sterile propylene vials (Pleion, Brazil), from a batch that had been previously tested for plastic toxicity to spermatic motility. Samples were collected following a 2- to 3-day period of absence of ejaculation, with a one-week interval between collections.

The macroscopic assessment, identical for all 3 seminal samples, was performed according to the criteria in the manual of World Health Organization (5). The following parameters were assessed: coagulation, liquefaction time, color, aspect, volume, viscosity and $\mathrm{pH}$.

The microscopic assessment was performed differently for each collected sample:

1) First seminal sample: after assessing the macroscopic parameters, the sample was homogenized by manually agitating the collecting vial and, with the aid of an automatic pipette (MLA, USA) a $5-\mu \mathrm{L}$ drop of semen was placed in the center of a Horwell counting chamber (Arnold R. Horwell Limited, London). The drop was covered by a glass coverslip and observed under a common light microscope (Nikon, model Eclipse 200, Japan), at 400X magnification, in order to verify the presence of spermatozoa and round cells, as well as to assess spermatic motility. Similarly, this procedure was repeated with 2 additional $5-\mu \mathrm{L}$ aliquots of semen. In cases where spermatozoa were found, the spermatic concentration was determined through volumetric dilution associated with hematocytometry, using an optimized Neubauer chamber. Values were expressed in millions 


\section{Flow Chart}

Patients referred for evaluation with initial diagnosis of non-obstructive azoospermia

$$
\downarrow
$$

Investigation protocol for azoospermia

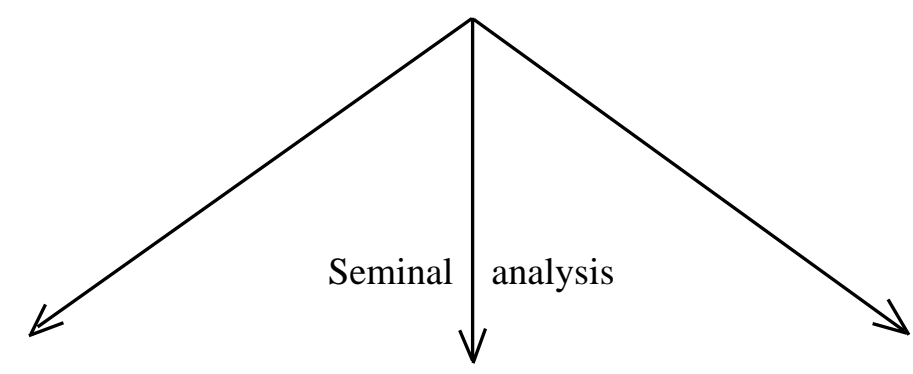

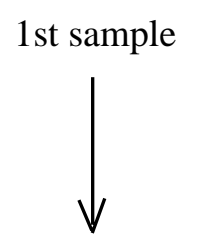

Horwell Fresh Sediment 2nd sample

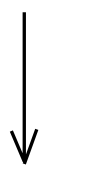

Horwell

Stained Sediment 3rd sample

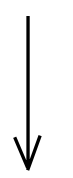

Horwell

Stained Percoll

Figure 1 - Flow chart for investigation of non-obstrictive azoospermia.

per milliliter (5). In cases where no spermatozoa were seen on the Horwell counting chamber, the entire seminal sample was transferred to a $15-\mathrm{mL}$ graded conic tube (Corning, reference 430791, USA). The tube was centrifuged at $1200 \mathrm{rpm}$ for 30 minutes. The supernatant was discarded, maintaining only $0.02 \mathrm{~mL}$ of sediment in the tube that was observed between glass slide and coverslip with a common light microscope, at 400X magnification, in order to verify presence or absence of spermatozoa.

2) Second seminal sample: using the Horwell chamber, the search for spermatozoa was performed similarly to the first seminal sample. In cases where spermatozoa were not seen in the Horwell chamber in the second sample, the entire seminal material was transferred to a $15-\mathrm{mL}$ graded conic tube (Corning, reference 430791, USA). The sample was centrifuged at $1200 \mathrm{rpm}$ for 30 minutes. The supernatant was discarded, maintaining only $0.02 \mathrm{~mL}$ of sediment in the tube, with the addition of $0.5 \mathrm{~mL}$ of Biggers, Whitten and Whittingham culture medium (BWW medium). A new centrifugation was performed with identical velocity and time. The supernatant was discarded and the sediment was used for preparing a smear on a clean and labeled glass slide. The slide containing the smear was left to dry at room temperature and, subsequently, was placed in an air stove (Fanem, model $002 \mathrm{CB}$, Brazil) at temperature of $36.5^{\circ} \mathrm{C}$ for 6 hours, so that complete fixation of the material would take place. Each slide was stained by the panoptic method, and the observation of spermatozoa and germ epithelial cells was performed through common light microscopy (Nikon, Microphot FXA, Japan) with 1250X magnification, under immersion (6). 
3) Third seminal sample: spermatozoa were initially searched in $35-\mu \mathrm{L}$ drops of semen, similarly to the procedure used for the first seminal sample. In this third sample, when no spermatozoa were observed on the Horwell chamber, the entire seminal material was transferred to a $15-\mathrm{mL}$ grade conic tube (Corning, reference 430791, USA). This material underwent centrifugation at $1200 \mathrm{rpm}$ for 30 minutes. The supernatant was discarded, the sediment was re-suspended in $1 \mathrm{~mL}$ of BWW culture medium and transferred to a $15-\mathrm{mL}$ graded conic tube, containing discontinuous Percoll ${ }^{\circledR}$ density gradient (Amershan, Pharmacia Biotech, reference 17-0891-01, Uppsala, Sweden) with 3 layers (45\%, 70\% and 90\%), with $1.5 \mathrm{~mL}$ of $45 \%$ layer, $1 \mathrm{~mL}$ of $70 \%$, and $1 \mathrm{~mL}$ of $90 \%$. The tube was centrifuged again at $1200 \mathrm{rpm}$ for 30 minutes. Each layer was aspirated separately, laid in a graded conic tube, containing $5 \mathrm{~mL}$ of culture medium (BWW) and centrifuged at $1200 \mathrm{rpm}$ for $10 \mathrm{~min}-$ utes. Following this period, the supernatant was discarded, maintaining $10 \mu \mathrm{L}$ of sediment in the tube, which were used for preparing a smear in a clean and labeled slide. The slides containing the smears from each layer were left to dry at room temperature and were subsequently placed in an air stove (Fanem, $002 \mathrm{CB}$, Brazil), at $36.5^{\circ} \mathrm{C}$ for 6 hours, so that fixation of the material could occur. Each slide was stained by the panoptic method. The observation of spermatozoa and germ epithelial cells was performed through common light microscopy (Nikon, Microphot FXA, Japan) with $1250 \mathrm{X}$ magnification under immersion (6).

\section{RESULTS}

The clinical and laboratorial data of the patients enrolled in this study are presented in Table-1.

After collection and analysis of the 1st seminal sample, of the 27 patients who participated in this study with presumed diagnosis of non-obstructive azoospermia, 3 presented spermatozoa during observation in the Horwell chamber (mean spermatozoa of 5,000/mL) and another patient presented spermatozoa on the fresh analysis of the seminal material following centrifugation. Thus, 4/27 (14.8\%) patients presented spermatozoa on the analysis of the 1 st semen sample performed at the laboratory.
The remaining 23 patients were referred for collection and analysis of the 2nd seminal sample according to the protocol established in this research. While no spermatozoon was identified with the Horwell chamber, 6 patients evidenced spermatozoa on the analysis of the stained sediment, totalizing $26.1 \%$ of patients with spermatozoa on the analysis of the 2nd seminal sample.

The remaining 17 patients underwent collection of the 3rd seminal sample. In these patients, no spermatozoon was detected with the Horwell chamber, neither in fresh sediment, in the sediment stained by panoptic method or in the separated and stained Percoll fractions. However, fractions stained by panoptic method revealed that $11 / 17(64.7 \%)$ patients presented young elements of the germ lineage, more specifically, spermatids. Data are summarized in Table-2.

\section{COMMENTS}

A patient with no relevant clinical antecedents, no abnormalities on the genital physical examination, spermiogram with volume equal or superior to $2 \mathrm{~mL}$ and absence of spermatozoa could be considered as having non-obstructive azoospermia (5). However, if this patient undergoes collection and analysis of a second seminal sample and some spermatozoa are detected, how should this patient be considered? As someone with severe oligozoospermia and not non-obstructive azoospermia anymore? Having virtual azoospermia, as proposed by Tournaye et al. (7), in contrast with absolute azoospermia, when no spermatozoon is found in the ejaculate or in the post-centrifuged sediment. This condition should be considered as cryptozoospermia or intermittent azoospermia as other authors advocate (8). Could it be only a categorization issue, a question of semantics, or simply a motive for debates among experts? The slightest such difference could be, would it bring relevant clinical implications?

Considering the history and progress achieved in the field of reproductive medicine during the 90 s, we must differentiate 2 periods: before and after 1992, when the technique of intracytoplasmic sperm injection (ICSI) was clinically applied for 
Table 1 - Clinical and laboratorial data from the 27 patients under study.

\begin{tabular}{|c|c|c|c|c|c|}
\hline \multirow[t]{2}{*}{$\mathbf{N}$} & \multirow{2}{*}{$\begin{array}{c}\text { Age } \\
\text { (years) }\end{array}$} & \multicolumn{2}{|c|}{ Testicular Volume (mL) } & \multirow{2}{*}{$\begin{array}{c}\text { FSH } \\
(\mathrm{mIU} / \mathrm{mL})\end{array}$} & \multirow{2}{*}{$\begin{array}{c}\text { Presence of } \\
\text { Spermatozoa }\end{array}$} \\
\hline & & Right & Left & & \\
\hline 1 & 33 & 10 & 12 & 18.4 & \\
\hline 2 & 29 & 20 & 20 & 5.6 & \\
\hline 3 & 18 & 9 & 8 & 44.6 & + \\
\hline 4 & 34 & 20 & 20 & 4.6 & \\
\hline 5 & 39 & 20 & 20 & 1.6 & + \\
\hline 6 & 39 & 13 & 12 & 60.1 & \\
\hline 7 & 43 & 13 & 13 & 11.7 & \\
\hline 8 & 42 & 13 & 12 & 9.0 & + \\
\hline 9 & 24 & 13 & 13 & 6.8 & + \\
\hline 10 & 37 & 25 & 15 & 9.0 & \\
\hline 11 & 46 & 10 & 10 & 17.7 & \\
\hline 12 & 42 & 13 & 13 & 40.8 & \\
\hline 13 & 37 & 25 & 18 & 7.9 & + \\
\hline 14 & 32 & 25 & 25 & 1.0 & \\
\hline 15 & 33 & 12 & 12 & 44.5 & + \\
\hline 16 & 33 & 12 & 12 & 25.6 & \\
\hline 17 & 38 & 12 & 12 & 16.1 & + \\
\hline 18 & 33 & 12 & 12 & 28.7 & + \\
\hline 19 & 48 & 15 & 10 & 19.1 & \\
\hline 20 & 34 & 15 & 13 & 29.0 & \\
\hline 21 & 33 & 20 & 20 & 10.8 & \\
\hline 22 & 34 & 17 & 17 & 16.0 & \\
\hline 23 & 35 & 15 & 15 & 4.2 & + \\
\hline 24 & 33 & 18 & 16 & 16.0 & + \\
\hline 25 & 34 & 15 & 12 & 26.0 & \\
\hline 26 & 37 & 25 & 25 & 7.6 & \\
\hline 27 & 30 & 20 & 20 & 11.6 & \\
\hline
\end{tabular}

the first time, leading to the birth of the first child generated by this kind of biotechnology (9). Until 1991, under a clinical, therapeutic and prognostic perspective, the discussion between non-obstructive azoospermia and severe oligozoospermia was meaningless, and this difference had more academic than clinical interest. On the other hand, with the advent of ICSI, this difference gains a new dimension, because non-obstructive azoospermic patients could not be genetic fathers, while patients with severe oligozoospermia could constitute a family with their own genes (10).

Table 2 - Summary of laboratory results from patients under study.

\begin{tabular}{ccc}
\hline $\begin{array}{c}\text { 1st sample }(27 \mathrm{pts}) \\
\text { Presence of Spermatozoa }\end{array}$ & $\begin{array}{c}\text { 2nd sample }(23 \mathrm{pts}) \\
\text { Presence of Spermatozoa }\end{array}$ & $\begin{array}{c}\text { 3rd sample }(17 \mathrm{pts}) \\
\text { Presence of Spermatids }\end{array}$ \\
\hline Horwell Method, $\mathrm{N}=3$ & Horwell Method, $\mathrm{N}=0$ & Horwell Method, $\mathrm{N}=0$ \\
Fresh Sediment, $\mathrm{N}=1$ & Stained Sediment, $\mathrm{N}=6$ & Stained Percoll, $\mathrm{N}=11$ \\
Total $=4 / 27(14.8 \%)$ & Total $=6 / 23(26.1 \%)$ & Total $=11 / 17(64.7 \%)$ \\
\hline
\end{tabular}


Due to this dramatic change, the concept of azoospermia itself, as issued by the manual of the World Health Organization, should be reconsidered. When should we consider a patient as azoospermic? Searching for spermatozoa in a single seminal sample or in several samples? Should we always consider fresh research following centrifugation? Which time and centrifuge force applied to the semen contained in the tube would reassure us about the final result? Would the laboratories be prepared for and warned about these differences and their consequences? Additionally, would the patients and physicians themselves be prepared? All this serves to promote greater reflection about a spermiogram report showing previous azoospermia and seminal volume over $2 \mathrm{~mL}$.

How can we be sure if a patient with nonobstructive azoospermia has or not spermatozoa in the ejaculate or within his testes? We have recently learned, through several published works, that the patient's age, testicular volume and serum FSH level are not reliable parameters for predicting, in last instance, what happens inside the testis $(11,12)$.

Apparently, the most reliable parameter is the histopathological examination of testicular fragments obtained from testicular biopsy (13). Despite having higher predictive value, it would constrain the patient to undergo, in practical terms, at least 2 biopsies, one diagnostic and another therapeutic, with all risks and inconveniences derived from these procedures (14).

Many investigators have searched the answer through a more attentive analysis of germ elements present in the ejaculate, thus considering not only the presence of spermatozoa, but immature elements of the germ lineage as well $(15,16)$. Through an exhaustive research in the seminal fluid, the investigator tries to detect any haploid cell that could better reflect what is happening inside the seminiferous tubules. In this work, we addressed these 2 aspects, that is, the presence of spermatozoa or young cells (spermatids) in the ejaculate of patients considered as having nonobstructive azoospermia, with prognostic purposes, considering the ICSI technique.

It is worth to stress that all 27 patients enrolled in this study had been initially diagnosed with non-obstructive azoospermia, as confirmed by a spermiogram performed at other centers. The first seminal analysis performed at our laboratory revealed already 4 patients with spermatozoa. In the second sample, other 6 patients were added, revealing that $10 / 27(37 \%)$ could no longer be considered azoospermic strictly speaking. On the other hand, among the 17 patients who underwent the third sample, 11 did not shoe spermatozoa, but had spermatids. The importance of finding such cells has not been completely established yet. However, since the maturation stop is considered infrequent during this stage, it allow us to assume that some areas inside the testes can present further differentiated elements, such as elongated spermatids, or even islets containing spermatozoa $(14,17)$. Even with experimental character, spermatids might be injected into oocytes, producing pre-embryos and pregnancy, as the literature has already shown (4).

The present data allow us to conclude that one routine seminal analysis is not enough to establish the diagnosis of non-obstructive azoospermia. Additionally, in clinical situations where the initial diagnosis is non-obstructive azoospermia, the analysis of the centrifuged sediment can have relevant clinical consequences. In this study, the majority of patients with initial diagnosis of non-obstructive azoospermia presented at least round spermatids in the ejaculate $(64.7 \%)$.

\section{REFERENCES}

1. Sigman M, Lipshultz LI, Howards SS: Evaluation of the Subfertile Male. In: Lipshultz LI \& Howards SS (eds.), Infertility in the Male. St. Louis, Mosby, 3ed. 1997; pp. 173-93.

2. Tesarik J, Mendoza C, Testart J: Viable embryos from injection of round spermatids into oocytes. N Engl J Med. 1995; 333: 525.

3. Fishel S, Green S, Hunter A, Lisi F, Rinaldi L, Lisi R, et al.: Human fertilization with round and elongated spermatids. Hum Reprod. 1997; 12: 336-40.

4. Cremades N, Bernabeu R, Barros A, Sousa M: In-vitro maturation of round spermatids using co-culture on Vero cells. Hum Reprod. 1999; 14: 1287-93.

5. World Health Organization - WHO Laboratory Manual for the Examination of Human Semen and Sperm-Cer- 
vical Mucus Interaction, 4th ed. Cambridge, Cambridge University Press. 1999.

6. Kruger TF, Menkveld R, Stander FS, Lombard CJ, Van der Merwe JP, van Zyl JA, et al.: Sperm morphologic features as a prognostic factor in in vitro fertilization. Fertil Steril. 1986; 46: 1118-23.

7. Tournaye H, Camus M, Goossens A, Liu J, Nagy P, Silber S, et al.: Recent concepts in the management of infertility because of non-obstructive azoospermia. Hum Reprod. 1995; 10: 115-9.

8. Mortimer D: Practical Laboratory Andrology. New York, Oxford University Press. 1994; pp. 3-12.

9. Palermo G, Joris H, Devroey P, Van Steirteghem AC: Pregnancies after intracytoplasmic injection of single spermatozoon into an oocyte. Lancet. 1992; 340: 17-8.

10. Tournaye H, Liu J, Nagy PZ, Camus M, Goossens A, Silber S, et al.: Correlation between testicular histology and outcome after intracytoplasmic sperm injection using testicular spermatozoa. Hum Reprod. 1996; 11: $127-32$.

11. Hauser R, Temple-Smith PD, Southwick GJ, de Kretser D: Fertility in cases of hypergonadotropic azoospermia. Fertil Steril. 1995; 63: 631-6.

12. Tournaye H, Verheyen G, Nagy P, Ubaldi F, Goossens A, Silber S, et al.: Are there any predictive factors for successful testicular sperm recovery in azoospermic patients? Hum Reprod. 1997; 12: 80-6.

13. Kahraman S, Yakin K, Samli M, Vanlioglu F, Karlikaya G, Sertyel S, et al.: A comparative study of three techniques for the analysis of sperm recovery: touch-print cytology, wet preparation, and testicular histopathology. J Assist Reprod Genet. 2001; 18: 35763.

14. Ezeh UI, Martin M, Cooke ID, Moore HD: Correlation of testicular pathology and sperm extraction in azoospermic men with ejaculated spermatids detected by immunofluorescent localization. Hum Reprod. 1998; 13: 3061-5.

15. Angelopoulos T, Krey L, McCullough A, Adler A, Grifo JA: A simple and objective approach to identifying human round spermatids. Hum Reprod. 1997; 12: 2208-16.

16. Gandini L, Lenzi A, Lombardo F, Pacifici R, Dondero F: Immature germ cell separation using a modified discontinuous Percoll gradient technique in human semen. Hum Reprod. 1999; 14: 1022-7.

17. Ezeh UI, Taub NA, Moore HD, Cooke ID: Establishment of predictive variables associated with testicular sperm retrieval in men with non-obstructive azoospermia. Hum Reprod. 1999; 14: 1005-12.

Received: September 12, 2004

Accepted after revision: January 4, 2005

\author{
Correspondence address: \\ Dr. Deborah M. Spaine \\ Rua Leandro Dupret, 204 / 43 \\ 04025-010, São Paulo, SP, Brazil \\ Fax: + 5511 5573-0014 \\ E-mail: debnine@terra.com.br
}

